\title{
Herpes simplex infection and stroke
}

T. Kherkheulidze ${ }^{1}$, N. Khizanishvili ${ }^{2}$, B. Tsikarishvili ${ }^{1}$, N. Kvirkvelia ${ }^{3}$, T. Losaberidze $^{2}$, E. Devidze 2 , S. Ingorokva 1 , M. Beridze²

Abstract WSC180818
${ }^{1}$ Tbilisi State Medical University HTMC Clinic, Neurology/Neurosurgery, Tbilisi, Georgia. ${ }^{2}$ The First University Clinic of Tbilisi State Medical University, Neurology, Tbilisi, Georgia. ${ }^{3}$ Petre Sarajishvili Institute of Neurology, Neurology, Tbilisi, Georgia.

Background: Infective vasculities can cause the inflammation of cerebral vessels and high coagulation state that can become the risk for ischemic stroke

\section{Patients and Methods:}

Totall 75 ischemic stroke patients investigated. 40 male/35 female, aged 50-75 with anamnesis of herpes simplex infection during 3 months previously of stroke have been investigated. All major risk factors of stroke (Atherosclerosis, diabetes mellitus,hypertension,atrial fibrillation,cardiac diseases smoking,alcohol abuse) were researched. Stroke severity assessed by NIHSS scale. Brain MRI (1.5 Tesla) was done. Blood investigated for antinuclear antibodies (ANA) and Antineutrophil cytoplasmic antibody (ANCA) by Enzymelinkedimmunosorbent Assay (ELISA) method,C reactive protein and coagulation, serology for herpes simplex virus (HSV 1/2). Statistics were done by SPSS -14.0. Pearson correlation and Multivariate logistic regression (entered stepwise model) were performed.

Relation of blood ANA and ANCA with blood INR (Linear regression analysis) ANA

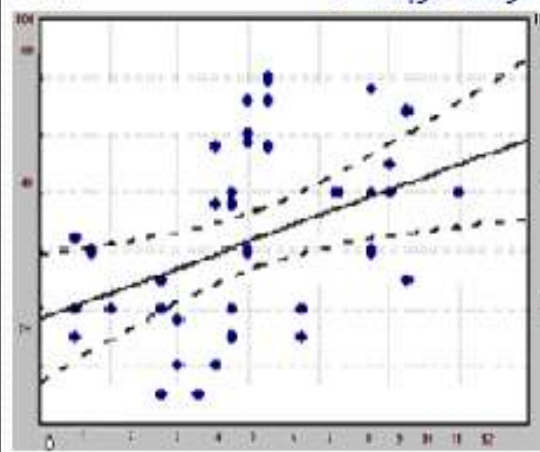
$\mathrm{R}=+0.45 \quad \mathrm{P}<0.05$ ANCA $\mathrm{R}=+0.27 \mathrm{P}<0.05$

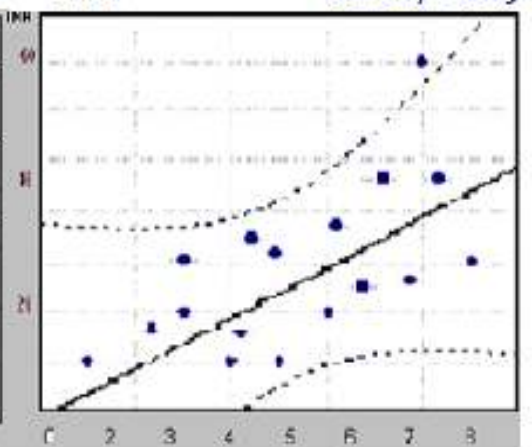

Positive correlation was found between blood ANA and ANCA levels with Blood INR levels in stroke patients $(R=+0.45$ and $R=+0.27$ respectively, $P<0.05)$

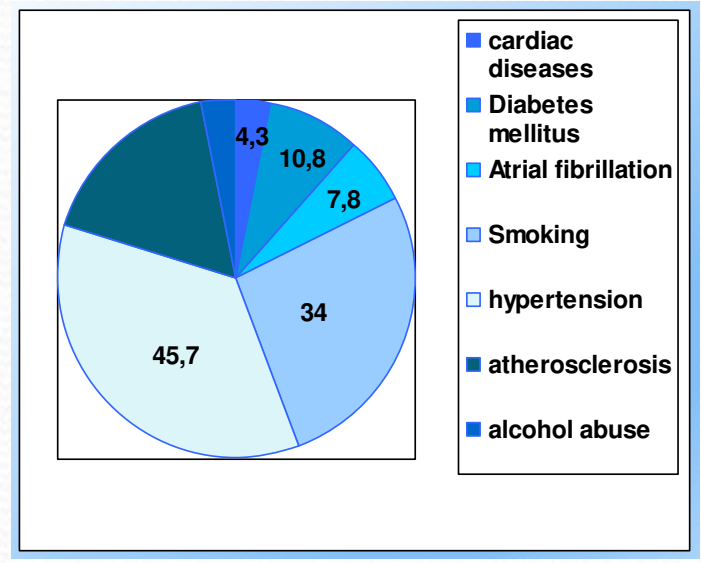

\section{Results:}

All 75 patients showed positive serology for herpes infection

48 positive for $\mathrm{HSV} 1$

27 patients -for $\mathrm{HSV} 2$.

Blood C reactive protein was elevated in all patients $(18 \pm 5.1)$,

$\checkmark$ blood ANA was elevated in $75 \%$ of patients (11.o U士4.2)

$\checkmark$ Anti PR 3 (c-ANCA) in $50 \%$ of patients (o.97U士0.05).

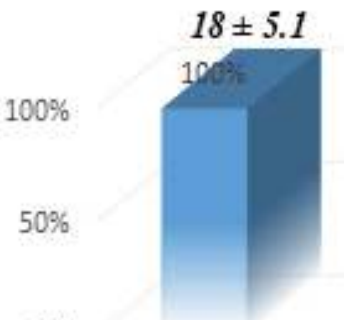

Blood C reactive proten

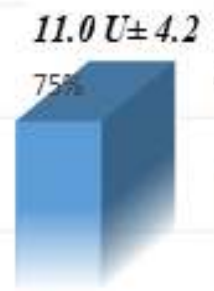

blood ANA
$0.97 U \pm 0.05$

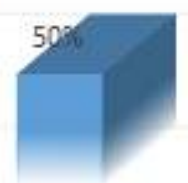

Anti PR 3 (cANCA

Multivariate logistic regression revealed the significance of blood high ANA and ANCA levels in conjunction with diabetes for severity of stroke measured by NIHSS (p<0.05).

There was not significant correlation between ANA and ANCA and ischemic lesion size on MRI scans

Conclusion: According to present research Herpes simplex virus can cause the infectious vasculitis in several immunocompromised patients and by increasing the inflammatory reactions might help to the ischemic stroke development. 